\title{
Yet Another Proof of the Cascade Decomposition Theorem for Finite Automata: Correction
}

by

\author{
Paul Zeiger
}

Dr. Jurg Nievergelt of the University of Illinois has pointed out that Method II can be blocked in a way not covered in lines 9 and 10 of page 227 (Math. Systems Theory 1 (1967), 225-228): if $\operatorname{sgrp} A$ consists entirely of permutations and resets, then $T$ will be the ideal of resets and $V$ the group of units; Method II will then produce a first component that is permutation-reset, and hence no simpler than the original automaton. To salvage the proof we eliminate the resets from this first component by modifying the method as follows: Let st $B_{1}=V$ instead of $T$, then whenever $u$ is in $T$, let $p^{\prime}=p$ (instead of $\underline{u}$ ), and $r^{\prime}=p^{-1}$ (the state to which $\underline{u}$ resets), instead of $p(r)$. This method (call it IIA) then suffices to decompose a permutationreset automaton into a permutation automaton followed by a reset automaton; since the methods as originally stated bring an arbitrary automaton to a cascade of permutation-reset automata, Method IIA finishes the job.

\section{Invariance for Ordinary Differential Equations: Correction}

\author{
by
}

JAMES A. Yorke

We wish to make some corrections to our recent paper*. At the end of the statement of Theorem 2.3 on p. 357 , add the words:

“. . if $g(x)$ is subtangential to $V$ for all $x \in \Lambda$." On p. 360, line 4 , insert $\bar{J} \cap \bar{I}$ between the words "so" and "is". On p. 361, line 16, add the phrase ". . when $\varphi=w(\varphi(t), u(t))$ almost everywhere" after the word "Then", and, in the last display, replace the first equality sign by $\leqq$, noting (1) that this display is valid for almost all $\tau$, and (2) that it now follows that $V(\varphi(t), t)$ is an absolutely continuous non-increasing function.

On p. 363, line 6 , the condition that $t_{n} \rightarrow \infty$ is needed, and in lines 19-20, $t+t_{n}$ should be replaced by $t+t_{n_{i}}$. On p. 368 , the reference should be to Theorem 3.3, not Theorem 3.6.

*Math. Systems Theory 1 (1967), 353-372. 